\title{
Estudio multicéntrico de la vigilancia de la susceptibilidad in vitro a tigeciclina en Santiago de Chile
}

\author{
Patricia García C., Chrystal Juliet L., Alejandra Fernández V., Marcela San Martín S., Marcela Cifuentes D., \\ Lorena Porte T., Stephanie Braun J., Loriana Castillo D., Maggie Vechiola H., Cecilia Tapia P., \\ Andrea Sakurada Z., Leonardo Chanqueo C., Marusella Lam E., Mónica Espinoza P. y Daniel Curcio F.
}

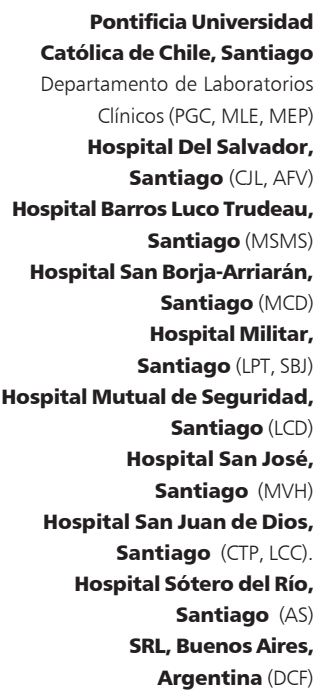

Este estudio fue financiado por Laboratorios Wyeth Inc. y corresponde a los datos chilenos de un trabajo colaborativo con Argentina.

Recibido: 30 de junio 2008 Aceptado: 21 de enero 2009

Correspondencia a:

Patricia García C. pgarcia@med.puc.cl

\section{Introducción}

T igeciclina (Tygacil® ${ }^{\circledR}$, Wyeth Pharmaceuticals) es un antimicrobiano que pertenece a la familia de las glicilciclinas, derivada de la molécula de minociclina, emparentada con las tetraciclinas, que presenta al igual que éstas, un amplio espectro antibacteriano. El desarrollo de este derivado, que incorpora el radical N,N-dimetilglicilamido en la posición 9 de la minociclina, evita los dos principales mecanismos de resistencia a tetraciclinas: la protección ribosomal (genes tetM o tetO), y los condicionados por bombas de eflujo (como tetA-tetE de enterobacterias y Acinetobacter sp y tetK en Staphylococcus sp) y ha mejorado el espectro de acción antibacteriana ${ }^{1,2}$.

El mecanismo de acción, al igual que las tetraciclinas, es la inhibición de la síntesis proteica por unión a la sub-unidad ribosomal 30S (uniéndose en una orientación diferente que las tetraciclinas), donde bloquea la entrada del ARN-t al ribosoma ${ }^{3}$.
Su comercialización fue aprobada por la Food and Drug Administration (FDA) en junio de 2005, y posteriormente, por la European Medicines Agency (EMEA) en abril de 2006, para el tratamiento empírico, en monoterapia, de infecciones intra-abdominales, de piel y tejidos blandos, tanto en el hospital como las adquiridas en la comunidad, incluyendo apendicitis complicadas, perforaciones y abscesos intra-abdominales, infecciones profundas de tejidos blandos, quemaduras y úlceras infectadas ${ }^{4-6}$.

Tigeciclina representa un gran avance en la terapia antimicrobiana ya que es activa frente a bacterias resistentes grampositivas y gramnegativas. La actividad de tigeciclina se ha estudiado ampliamente, tanto in vitro como in vivo, sobre una gran variedad de patógenos ${ }^{7-13}$. Entre los microorganismos grampositivos aerobios es activa sobre Streptococcus pneumoniae, incluyendo las cepas resistentes a penicilina; Staphylococcus aureus (sensibles y resistentes a meticilina, VISA y VRSA), Staphylococcus epidermidis 
(sensibles y resistentes a meticilina), Streptococcus agalactiae, Streptococcus pyogenes y Streptococcus grupo anginosus ( $S$. anginosus, $S$. intermedius y $S$. constellatus), Enterococcus faecalis, Enterococcus faecium, Enterococcus avium, Enterococcus casseliflavus y Enterococcus gallinarum (sensibles y resistentes a vancomicina) y Listeria monocytogenes.

La actividad sobre bacterias gramnegativas incluye a Escherichia coli, Klebsiella pneumoniae, Klebsiella oxytoca, Enterobacter cloacae, Enterobacter sp, Citrobacter freundii, Citrobacter sp, Serratia marcescens, Serratia sp y Aeromonas hydrophila, con buena actividad sobre las enterobacterias productoras de $\beta$-lactamasas de espectro expandido (BLEE). También es activa sobre Haemophilus influenzae, Moraxella catarrhalis, Pasteurella multocida, Eikenella corrodens y algunos no fermentadores como Acinetobacter baumannii, Stenotrophomonas maltophilia y Burkholderia cepacia. La actividad sobre Pseudomonas aeruginosa y sobre especies de Proteus y Providencia es limitada presentando $\mathrm{CIM}_{90}>8 \mu \mathrm{g} / \mathrm{ml}$.

Existen los siguientes puntos de corte aprobados por la FDA para microdilución en caldo: categoría sensible para $S$. aureus (sensible o resistente a meticilina) con CIM $<0,5 \mu \mathrm{g} / \mathrm{ml}$, categoría sensible para Streptococcus grupos A, B, C y G y para E. faecalis con CIM $<0,25 \mu \mathrm{g} / \mathrm{ml}$. Enterobacterias, A. baumannii y Proteus sp, Providencia sp y Morganella sp se consideran sensibles si la CIM es $<2 \mu \mathrm{g} / \mathrm{ml}$, intermedias si la CIM es de $4 \mu \mathrm{g} / \mathrm{ml}$ y resistentes si la CIM es $\geq 8 \mu \mathrm{g} / \mathrm{ml}^{14}$. Para el método de difusión en agar se utilizan discos con una carga de $15 \mu \mathrm{g}$ de tigeciclina ${ }^{15}$. En algunas cepas de Klebsiella sp, Enterobacter sp, Proteus sp y Morganella sp se han descrito CIMs a tigeciclina más elevadas, explicado por la presencia de algunas bombas de eflujo complejas ${ }^{16-18}$.

Dado que en Chile se estaban efectuando estudios clínicos fase 3, era necesario conocer el comportamiento in vitro de cepas chilenas causantes de infección. Con la colaboración de Wyeth-Argentina, se diseñó un estudio multicéntrico cuyos objetivos fueron conocer la sensibilidad in vitro a tigeciclina mediante el método de difusión en agar, determinar los patrones de susceptibilidad bacteriana a tigeciclina en diferentes hospitales de Santiago y comparar las cifras de susceptibilidad a tigeciclina con la de otros agentes antimicrobianos.

\section{Material y Método}

El estudio fue diseñado para realizar la vigilancia de la susceptibilidad in vitro de cepas chilenas en nueve hospitales de Santiago de Chile, con una duración de
14 meses (desde octubre de 2005 a diciembre de 2006). Se incluyeron las cepas obtenidas de pacientes hospitalizados con infecciones clínicamente significativas, aisladas en cualquier tipo de muestra exceptuando orina. Se consideró sólo una cepa por paciente.

Cada centro determinó la susceptibilidad a tigeciclina a 20 aislados de 20 muestras clínicas procesadas mensualmente, por el método de difusión en agar con discos de $15 \mu \mathrm{g}$ de tigeciclina provenientes del mismo lote. Las cepas intermedias y resistentes a tigeciclina más $10 \%$ de las cepas susceptibles (dos cepas por centro al mes) fueron derivadas al laboratorio de microbiología de la Pontificia Universidad Católica de Chile (centro coordinador). Además se derivaron las planillas con todos los datos individuales de los pacientes y los resultados de los datos locales obtenidos por difusión.

El laboratorio de microbiología de la Universidad Católica realizó el re-testeo de las cepas susceptibles mediante técnica de difusión. Para la evaluación de las cepas intermedias y resistentes se estudió difusión con discos y microdilución en caldo (JustOne, TREK ${ }^{\circledR}$ ). Se definió cepas concordantes si la valoración de la susceptibilidad (susceptible, intermedio y resistente) eran exactamente igual entre ambos métodos probados. El control de calidad de los discos se realizó en forma semanal y de los paneles de microdilución en caldo cada vez que éstos eran utilizados. Las cepas discordantes fueron congeladas a $-80^{\circ} \mathrm{C}$. Para el análisis final de los datos, se completó la base de datos enviada desde Argentina, en un programa Access.

Para la categorización de las cepas se utilizaron los puntos de corte recomendados por la FDA (Tabla 1) y para el control de calidad los recomendados por el CLSI (Tabla 2) durante $2006^{19}$.

\begin{tabular}{|c|c|c|c|c|c|c|}
\hline \multirow[t]{2}{*}{ Microorganismo } & \multicolumn{3}{|c|}{$\begin{array}{l}\text { Difusión** } \\
\text { (mm) }\end{array}$} & \multicolumn{3}{|c|}{$\begin{array}{c}\text { Dilución CIM*** } \\
(\mu \mathrm{g}(\mathrm{mL})\end{array}$} \\
\hline & $\mathbf{S}$ & $\mathbf{I}$ & $\mathbf{R}$ & $\mathbf{S}$ & I & $\mathbf{R}$ \\
\hline Staphylococcus aureus & $\geq 19$ & - & - & $\leq 0,5$ & - & - \\
\hline Enterococcus faecalis & $\geq 19$ & - & - & $\leq 0,25$ & - & - \\
\hline Enterobacterias & $\geq 19$ & $15-18$ & $\leq 14$ & $\leq 2$ & 4 & $\geq 8$ \\
\hline Proteus sp, Providencia sp, Morganella sp & $\geq 23$ & $20-22$ & $\leq 19$ & $\leq 2$ & 4 & $\geq 8$ \\
\hline \multicolumn{7}{|c|}{$\begin{array}{l}\mathrm{S}=\text { sensible, } \mathrm{I}=\text { intermedia, } \mathrm{R}=\text { resistente } \\
\text { *No hay puntos de corte recomendados por CLSI. } \\
\text { **Discos con } 15 \mu \mathrm{g} \text {. } \\
\text { *** Método de referencia: microdilución en caldo permite establecer CIM (Concentración } \\
\text { inhibitoria mínima). }\end{array}$} \\
\hline
\end{tabular}




\begin{tabular}{|c|c|c|c|}
\hline Cepa ATCC & & $\begin{array}{l}\text { Difusión (mm) } \\
\text { Discos } 15 \text { ug }\end{array}$ & $\begin{array}{c}\text { Dilución }(\mu \mathrm{g} / \mathrm{mL}) \\
\text { CIM }\end{array}$ \\
\hline Escherichia coli & 25.922 & $20-27$ & $0,03-0,25$ \\
\hline Staphylococcus aureus & 25.923 & $20-25$ & - \\
\hline Streptoccus pneumoniae & 49.619 & $23-29$ & $0,015-0,12$ \\
\hline Pseudomonas aeruginosa & 27.853 & $9-13$ & - \\
\hline Enterococcus faecalis & 29.212 & - & $0,03-0,12$ \\
\hline Staphylococcus aureus & 29.213 & - & $0,03-0,25$ \\
\hline
\end{tabular}

Figura 1. Diseño del estudio de vigilancia de la susceptibilidad in vitro a tigeciclina y número de cepas estudiadas.

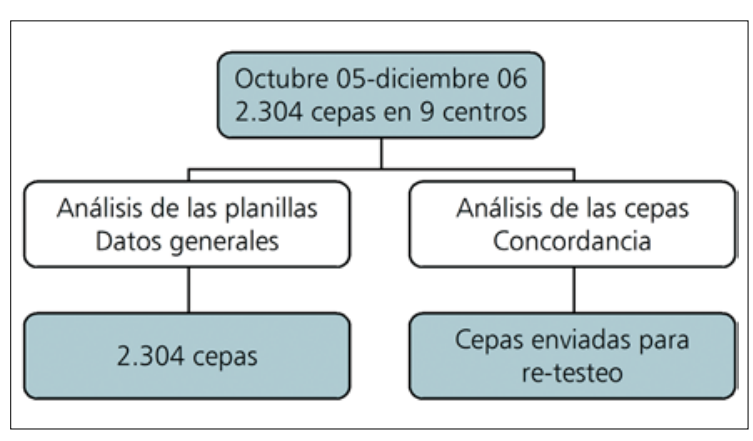

\begin{tabular}{|c|c|c|}
\hline Microorganismos & $\mathbf{n}$ & $\%$ \\
\hline $\begin{array}{l}\text { Enterobacterias: E. coli - K. pneumoniae - } \\
\text { Serratia sp - Enterobacter sp - Citrobacter sp - } \\
\text { Salmonella sp Shigella sp - Hafnia sp }\end{array}$ & 1.008 & 44 \\
\hline Staphylococcus sp & 694 & 30 \\
\hline Acinetobacter baumannii & 208 & 9 \\
\hline Enterococcus sp & 109 & 4,7 \\
\hline Streptococcus sp & 108 & 4,7 \\
\hline Proteus sp - Providencia sp - Morganella sp & 84 & 3,6 \\
\hline Pseudomonas aeruginosa & 51 & 2,2 \\
\hline $\begin{array}{l}\text { Otros BNF (Stenotrophomonas sp - } \\
\text { Burkholderia sp) }\end{array}$ & 17 & 0,7 \\
\hline Otros géneros & 18 & 0,7 \\
\hline No consignado & 7 & 0,4 \\
\hline Total & 2.304 & 100 \\
\hline
\end{tabular}

Tabla 3. Distribución porcentual de los tipos de muestras de origen de las cepas estudiadas

\begin{tabular}{|lcc|}
\hline Tipo de muestra & n & \% \\
\hline Sangre & 1.047 & 45 \\
Otras & 415 & 18 \\
\hline Herida & 216 & 9 \\
\hline Otros líquidos (punción) & 194 & 8 \\
\hline Herida quirúrgica & 178 & 7,5 \\
\hline Expectoración & 86 & 5 \\
\hline Biopsia & 44 & 2 \\
\hline LBA & 31 & 1,5 \\
\hline Catéter & 28 & 1,2 \\
\hline Hisopado rectal & 21 & 1 \\
\hline No consignada & 14 & 0,6 \\
\hline LCR & 13 & 0,5 \\
\hline Drenaje & 12 & 0,5 \\
\hline Deposición & 5 & 0,2 \\
\hline Total & 2.304 & 100 \\
\hline
\end{tabular}

\section{Resultados}

En el período de estudio se incluyeron 2.304 cepas provenientes de los nueve centros hospitalarios participantes de Santiago de Chile. El análisis de los datos generales se efectuó considerando el total de las cepas. Además, se realizó un análisis de las cepas enviadas para re-testeo con el fin de evaluar concordancia entre los centros participantes y el centro coordinador (Figura 1).

La mayoría (45\%) de las muestras desde donde se aislaron las cepas en estudio fueron hemocultivos, es decir, correspondían a infecciones sistémicas (Tabla 3).

Distribución de las bacterias aisladas (Tabla 4). Un $44 \%$ correspondió a Enterobacterias, excluyendo Proteus sp, Providencia sp y Morganella sp, que sólo representaron 3,6\% de los microorganismos estudiados. Un $39,4 \%$ de las cepas fueron cocáceas grampositivas (Staphylococcus sp, Enterococcus sp y Streptococcus sp). Un 9\% de las bacterias estudiadas correspondieron a $A$. baumannii y sólo $2,2 \%$ a $P$. aeruginosa.

Susceptibilidad a tigeciclina por difusión en agar, categorizada por género bacteriano (Tabla 5). El 100\% de Streptococcus sp, Enterococcus sp y E. coli fueron susceptibles a tigeciclina, al igual que $99,8 \%$ de Staphylococcus sp, $93 \%$ de Klebsiella sp y $80 \%$ de $A$. baumannii. En cambio, en el grupo Proteus sp, Provi- 
dencia sp y Morganella $\mathrm{sp}$, la susceptibilidad fue 4\% y en $P$. aeruginosa fue $2 \%$.

Resultado comparativo de tigeciclina versus otros antimicrobianos en Klebsiella sp y A. baumannii (Figuras 3 y 4). En Klebsiella sp resistente a cefotaxima (91 cepas), 95\% fueron sensibles a tigeciclina y en $A$. baumannii resistente a imipenem (50 cepas), 80\% fueron sensibles a tigeciclina.

\section{Concordancia y discordancia entre centros evaluados}

Con respecto a las cepas que fueron derivadas al centro coordinador para re-testeo por discos, todas las cepas enviadas como sensibles fueron confirmadas como tales ( $100 \%$ de concordancia) (Figura 2$)$. Sin embargo, de las 300 cepas enviadas como resistentes (101) o intermedias (199), hubo 218 cepas discordantes (73\%). De estas 218 cepas, hubo 162 catalogadas por los centros participantes como intermedias y que resultaron sensibles al ser evaluadas en el centro coordinador y 56 cepas consideradas resistentes por los hospitales que fueron catalogadas como susceptibles o intermedias en el centro coordinador (Tabla 6). Para definir las discordancias se tomó una muestra representativa de 59 cepas que se re-testearon por el método de referencia (microdilución en caldo). La mayoría de las cepas discordantes correspondieron a $\mathrm{A}$. baumannii (40\%), seguidos de K. pneumoniae (24\%) (Figura 5). De las 59 cepas re-testeadas, 47 habían sido catalogadas como resistentes por los centros. La microdilución en caldo determinó que 45 eran sensibles y que dos eran intermedias. Las 12 cepas restantes cata-

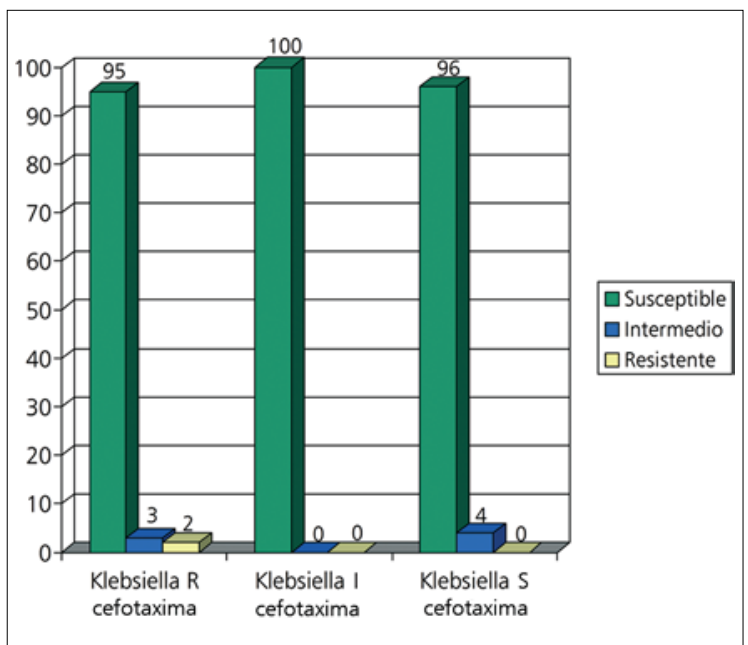

Figura 3. Comparación de la susceptibilidad a tigeciclina en cepas de Klebsiella sp susceptibles, intermedias y resistentes a cefotaxima, expresada en porcentaje.
Tabla 5. Susceptibilidad a tigeciclina de las cepas estudiadas mediante difusión en agar

\begin{tabular}{|c|c|c|c|c|}
\hline \multirow[t]{2}{*}{ Género bacteriano } & \multirow[b]{2}{*}{$\mathbf{n}$} & \multicolumn{3}{|c|}{ Tigeciclina } \\
\hline & & $\begin{array}{c}\mathbf{S} \\
(\%)\end{array}$ & $\begin{array}{c}1 \\
(\%)\end{array}$ & $\begin{array}{c}\mathbf{R} \\
(\%)\end{array}$ \\
\hline Staphylococcus aureus & 640 & 99,8 & 0,2 & 0 \\
\hline Staphylococcus coagulasa negativa & 54 & 100 & 0 & 0 \\
\hline Escherichia coli & 506 & 100 & 0 & 0 \\
\hline Klebsiella sp & 305 & 92,8 & 6,8 & 0,4 \\
\hline Enterococcus sp & 109 & 100 & 0 & 0 \\
\hline Streptococcus sp & 108 & 100 & 0 & 0 \\
\hline Acinetobacter baumannii & 208 & 80 & 19,5 & 0,5 \\
\hline Proteus sp, Providencia sp, Morganella sp & 84 & 4 & 30 & 66 \\
\hline Pseudomonas sp & 51 & 2 & 4 & 94 \\
\hline
\end{tabular}

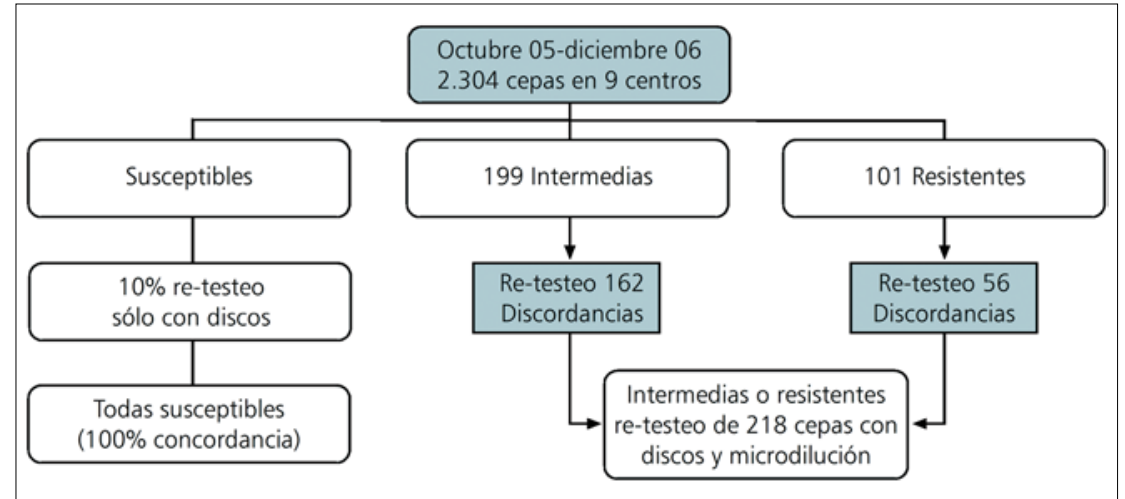

Figura 2. Análisis de las discordancias entre los centros y el laboratorio coordinador.

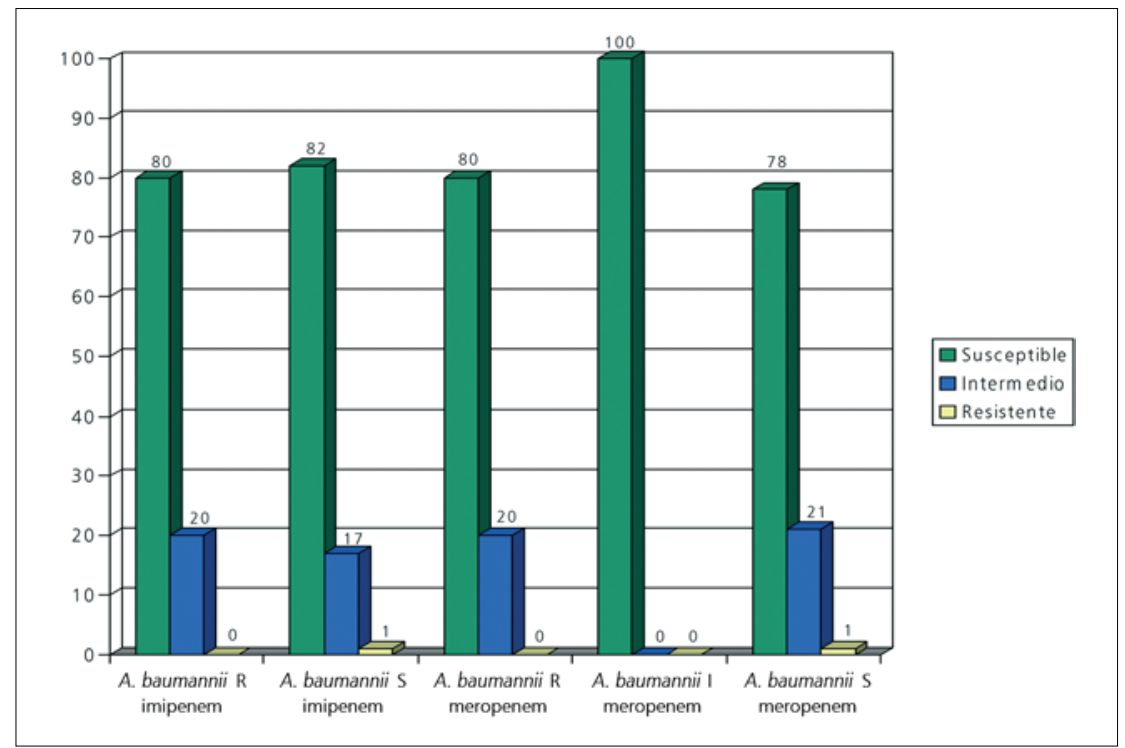

Figura 4. Comparación de la susceptibilidad a tigeciclina en cepas de Acinetobacter baumannii susceptibles, intermedias y resistentes a carbapenémicos, expresada en porcentaje. 


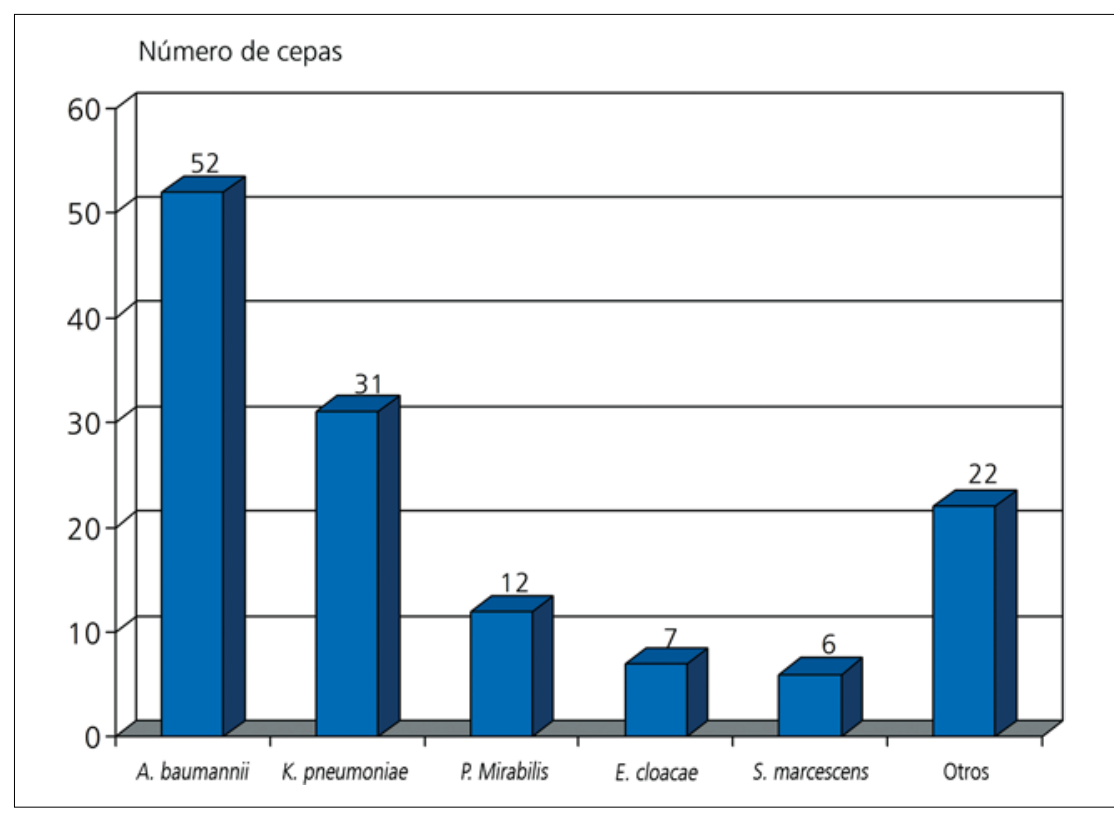

Figura 5. Distribución de las cepas discordantes por microorganismo.

\begin{tabular}{|c|c|c|c|c|}
\hline Información & de Centros & $\begin{array}{l}\text { Resultados Informados } \\
\text { Centro Coordinador }\end{array}$ & Discordancia & Error \\
\hline \multirow[t]{4}{*}{ Resistentes } & 101 & 45 resistentes & - & \\
\hline & & 31 sensibles & $30 \%$ & Mayor \\
\hline & & 25 intermedias & $25 \%$ & Menor \\
\hline & & Sub-Total: 56 & $55 \%$ & \\
\hline \multirow[t]{4}{*}{ Intermedias } & 199 & 37 intermedias & - & \\
\hline & & 157 sensibles & $79 \%$ & Menor \\
\hline & & 5 resistentes & $2,5 \%$ & Menor \\
\hline & & Sub-Total: 162 & $81,5 \%$ & \\
\hline Total & 300 & 218 discordancias & $73 \%$ & \\
\hline
\end{tabular}

logadas como intermedias por los centros, resultaron sensibles en la evaluación realizada por el centro coordinador.

\section{Discusión}

Los resultados de este estudio multicéntrico muestran que la susceptibilidad bacteriana a tigeciclina mediante el método de difusión en agar con discos de 15 $\mu \mathrm{g}$, en nueve hospitales de Santiago es alta en el grupo de las cocáceas grampositivas y en las enterobacterias tipo E. coli y K. pneumoniae. Esto es coincidente con lo reportado en la literatura científica, en que las $\mathrm{CIM}_{90}$ de S. aureus, Staphylococcus coagulasa negativa, Enterococcus sp y $S$. pneumoniae fluctúan entre $\geq 0,06$ y $0,5 \mu \mathrm{g} / \mathrm{mL}$ y, aproximadamente $95 \%$ de todas las Enterobacteriaceae son susceptibles a tigeciclina ${ }^{20}$. Como era esperable, en el grupo Proteus sp, Providencia sp y Morganella sp, la susceptibilidad fue menor al 5\%, probablemente debido a la presencia de bombas de eflujo del tipo AcrAB. Un fenómeno similar explicaría la alta resistencia observada en $P$. aeruginosa, en que los sistemas multibomba MexXY-OprM, MexABOprM, MexCD-OprJ y MexEF-OprN le confieren resistencia intrínseca a tigeciclina ${ }^{21}$.

Es importante destacar que la mayoría de las cepas incluidas en el estudio chileno fueron aisladas de hemocultivos, lo que comprueba la trascendencia clínica de los aislados. Además, la distribución por grupo bacteriano fue relativamente homogénea: $44 \%$ de las enterobacterias no Proteus sp, Providencia sp $y$ Morganella sp, 40\% de las cocáceas grampositivas y $12 \%$ de los bacilos gramnegativos no fermentadores, con un número importante de cepas de $A$. baumannii estudiadas, lo cual confiere mayor validez a los resultados obtenidos.

En el análisis comparativo de la susceptibilidad a tigeciclina en $A$. baumannii resistente a carbapenémicos y Klebsiella sp resistente a cefalosporinas de tercera generación, destaca que tigeciclina muestra una excelente susceptibilidad in vitro (80 y 95\%, respectivamente), tal como lo describe la literatura médica (Henwood et al. reportan actividad contra $80 \%$ de los aislados de Acinetobacter sp $)^{22-24}$. Este hecho permite considerar a tigeciclina como una buena alternativa de tratamiento en bacterias multiresistentes, que ya son una realidad en las Unidades de Cuidados Intensivos de nuestro país.

El análisis inicial de las concordancias permitió evidenciar una concordancia total con las cepas susceptibles; las discordancias se produjeron sólo en las cepas enviadas por los centros como intermedias y resistentes. Sin embargo, el porcentaje más alto correspondió a cepas informadas inicialmente como intermedias, en que los errores fueron menores. En las cepas derivadas al centro coordinador como resistentes hubo $30 \%$ de errores mayores, siendo todas éstas corroboradas como errores mayores por microdilución en caldo. La gran mayoría de estas discordancias se observaron en cepas de A. baumannii.

Debido a lo anterior, los autores consideran que la valoración de la susceptibilidad a tigeciclina mediante el método por difusión en agar requiere precaución para $A$. baumannii, más aún a la luz de la reciente descripción de bombas de eflujo ${ }^{25,26}$ en esta especie bacteriana. Además, está en estudio un cambio en los 
puntos de corte para difusión en agar en A. baumannii, pues investigaciones recientes muestran mejor correlación con el método de referencia ${ }^{27,28}$. No obstante, debe considerarse la eventual ocurrencia de errores primarios en el método por difusión que pueden ser explicados por la pérdida de potencia de los discos de tigeciclina en el tiempo y por factores inherentes al medio de cultivo ${ }^{29}$. Por esto, los autores recomiendan el re-testeo con microdilución en caldo frente a cepas de $A$. baumannii intermedias o resistentes a tigeciclina.

Este estudio multicéntrico permitió conocer las cifras locales de susceptibilidad a tigeciclina en cepas de pacientes hospitalizados con infecciones clínicamente significativas. La susceptibilidad observada es coincidente con la literatura científica. El método de difusión en agar requiere ser corroborado por técnica de dilución en cepas resistentes, especialmente $A$. baumannii.

\section{Resumen}

Para conocer la susceptibilidad a tigeciclina por difusión en agar en nueve hospitales de Santiago y comparar la susceptibilidad con otros antimicrobianos, se diseñó este estudio multicéntrico. Cada centro estudió 20 cepas mensualmente. Las intermedias, resistentes y $10 \%$ de las susceptibles se re-testearon y estudiaron por microdilución en caldo. Se incluyeron 2.304 cepas. Fueron susceptibles a tigeciclina Streptococcus sp (100\%), Enterococcus sp (100\%), E. coli (100\%), Staphylococcus sp (99,8\%), Klebsiella pneumoniae (93\%) y Acinetobacter baumannii (80\%). En Proteus, Providencia y Morganella la susceptibilidad fue 4\%. Klebsiella resistente a cefotaxima y Acinetobacter resistente a imipenem, 95\% y $80 \%$ fueron susceptibles a tigeciclina, respectivamente. La concordancia en cepas susceptibles y en las enviadas como resistentes o intermedias (A. baumannii) fue $100 \%$ y $27 \%$ respectivamente. El re-testeo confirmó que la mayoría eran susceptibles. Los patrones de susceptibilidad bacteriana muestran muy buena actividad in vitro a tigeciclina. La resistencia in vitro de $A$. baumannii por difusión en agar debe interpretarse con precaución.

\section{Referencias}

1.- Petersen P J, Jacobus N V, Weiss W J, Sum P E, Testa R T. In vitro and in vivo antibacterial activities of a novel glycylcycline, the 9-t-butylglycylamido derivative of minocycline (GAR-936). Antimicrob Agents Chemother 1999; 43: 738-44.

2.- Fluit A C, Florijn A, Verhoef J, Milatovic D. Presence of tetracycline resistance determinants and susceptibility to tigecycline and minocycline. Antimicrob Agents Chemother 2005; 49: 1636-8.

3.- Bauer G, Berens C, Projan S J, Hillen W. Comparison of tetracycline and tigecycline binding to ribosomes mapped by dimethylsulphate and drug-directed $\mathrm{Fe} 2+$ cleavage of 16S rRNA. J Antimicrob Chemother 2004 53: 592-9

4.- Pankey G A. Tigecycline. J Antimicrob Chemother 2005; 56: 470-80.

5.- Zinner S H. Overview of antibiotic use and resistance: setting the stage for tigecycline. Clin Infect Dis 2005; 41: S289-92.

6.- Noskin G A. Tigecycline: a new glycylcycline for treatment of serious infections. Clin Infect Dis 2005; 41: S303-14

7.- Fritsche $\mathrm{T}$ R, Kirby J T, Jones R N. In vitro activity of tigecycline (GAR-936) tested against 11,859 recent clinical isolates associated with community-acquired respiratory tract and Gram-positive cutaneous infections. Diagn Microbiol Infect Dis 2004; 49: 201-9.

8.- Sader H S, Jones R N, Dowzicky M J, Fritsche T R. Antimicrobial activity of tigecycline tested against nosocomial bacterial pathogens from patients hospitalized in the intensive care unit. Diagn Microbiol Infect Dis 2005; 52: 203-8.

9.- Sader H S, Jones R N, Stilwell M G, Dowzicky M J, Fritsche T R. Tigecycline activity tested against 26,474 bloodstream infection isolates: a collection from 6 continents. Diagn Microbiol Infect Dis 2005; 52: 181-6.

10.- Hoban D J, Bouchillon S K, Johnson B M, Johnson J L, Dowzicky M J. Tigecycline Evaluation and Surveillance Trial (TEST Program) Group. In vitro activity of tigecycline against 6792 Gram-negative and Gram-positive clinical isolates from the global Tigecycline Evaluation and Surveillance Trial (TEST Program, 2004). Diagn Microbiol Infect Dis 2005: 52; 21527.

11.- Gales A C, Jones R N, Andrade S S, Pereira A S, Sader H S. In vitro activity of tigecycline, a new glycylcycline, tested against 1,326 clinical bacterial strains isolated from Latin America. Braz J Infect Dis 2005: 9; 348-56.

12.- Bouchillon S K, Hoban D J, Johnson B M, Stevens T M, Dowzicky M J, Wu D H, et al.
In vitro evaluation of tigecycline and comparative agents in 3049 clinical isolates: 2001 to 2002. Diagn Microbiol Infect Dis 2005; 51: 291-5.

13.- Bradford P A, Weaver-Sands T, Petersen P J. In vitro activity of tigecycline against isolates from patients enrolled in phase 3 clinical trials of treatment for complicated skin and skin-structure infections and complicated intra-abdominal infections. Clin Infect Dis 2005; 41: S315-S332.

14.- Wyeth Pharmaceuticals Inc. Tygacil Product Insert. Philadelphia, PA, USA, 2007. http://www.tygacil.com

15.- Jones R N. Disk diffusion susceptibility test development for the new glycylcycline, GAR-936. Diagn Microbiol Infect Dis 1999; 35: 249-52.

16.- Ruzin A, Keeney D, Bradford P A. AcrAB efflux pump plays a role in decreased susceptibility to tigecycline in Morganella morganii. Antimicrob Agents Chemother 2005; 49: 791-3.

17.- Visalli M A, Murphy E, Projan S J, Bradford P A. AcrAB multidrug efflux pump is associated with reduced levels of susceptibility to tigecycline (GAR-936) in Proteus mirabilis. Antimicrob Agents Chemother 2003; 47: 665-9.

18.- Morosini M I, García-Castillo M, Coque T M, Valverde A, Novais A, Loza E, et al. Antibiotic co-resistance in extendedspectrum-beta-lactamase-producing 
Enterobacteriaceae and in vitro activity of tigecycline. Antimicrob Agents Chemother 2006; 50: 2695-9.

19.- Clinical and Laboratory Standards Institute. 2006. Performance standards for antimicrobial disk susceptibility tests; approved standard M2-A9, 9th ed. CLSI, Wayne, PA.

20.- Stein G E, Craig W A. Tigecycline: a critical analysis. Clin Infect Dis 2006; 43: 518-24.

21.- Dean C R, Visalli M A, Projan S J, Sum P E, Bradford P A. Efflux-mediated resistance to tigecycline (GAR-936) in Pseudomonas aeruginosa PAO1. Antimicrob Agents Chemother 2003; 47: 972-8.

22.- Henwood C J, Gatward T, Warner M, James D, Stockdale M W, Spence R P, et al. Antibiotic resistance among clinical isolates of Acinetobacter in the UK, and in vitro evaluation of tigecycline (GAR-936). J
Antimicrob Chemother 2002; 49: 479-87.

23.- Pachón-Ibáñez M E, Jiménez-Mejías M E, Pichardo C, Llanos A C, Pachón J. Activity of tigecycline (GAR-936) against Acinetobacter baumannii strains, including those resistant to imipenem. Antimicrob Agents Chemother 2004; 48: 4479-81.

24.- Halstead D C, Abid J, Dowzicky M J. Antimicrobial susceptibility among Acinetobacter calcoaceticus-baumannii complex and Enterobacteriaceae collected as part of the Tigecycline Evaluation and Surveillance Trial. J Infect 2007; 55: 49-57

25.- Peleg A Y, Adams J, Paterson D L. Tigecycline efflux as a mechanism for nonsusceptibility in Acinetobacter baumannii. Antimicrob Agents Chemother 2007; 51: 2065-9.

26.- Navon-Venezia S, Leavitt A, Carmeli Y. High tigecycline resistance in multidrug- resistant Acinetobacter baumannii. J Antimicrob Chemother 2007; 59: 772-4.

27.- Jones R N, Ferraro M J, Reller L B, Schreckenberger P C, Swenson J M, Sader H S. Multicenter studies of tigecycline disk diffusion susceptibility results for Acinetobacter spp. J Clin Microbiol 2007; 45: 227-30.

28.- Curcio D, Fernández F, Jones R N, Ferraro M J, Reller L B, Schreckenberger P $\mathrm{C}$, et al. Tigecycline disk diffusion breakpoints of Acinetobacter spp: a clinical point of view. J Clin Microbiol 2007; 45: 2095-6.

29.- Petersen P J, Bradford P A. Effect of medium age and supplementation with the biocatalytic oxygen-reducing reagent oxyrase on in vitro activities of tigecycline against recent clinical isolates. Antimicrob Agents Chemother 2005 49(9): 3910-8 